\title{
Higher Expression of Activation-induced Cytidine Deaminase Is Significantly As- sociated with Merkel Cell Polyomavirus-negative Merkel Cell Carcinomas
}

\author{
Michiko Matsushita,* Takeshi Iwasaki, $†$ Daisuke Nonaka,ł Satoshi Kuwamoto,§ Keiko Nagata,§ Masako Kato,§ \\ Yukisato Kitamura* and Kazuhiko Hayashi§ \\ *Department of Pathobiological Science and Technology, School of Health Science, Tottori University Faculty of Medicine, Yonago \\ 683-8503, Japan, †Department of Anatomic Pathology, Graduate School of Medical Sciences, Kyushu University, Fukuoka 812-8582, \\ Japan, $¥$ Department of Histopathology, The Christie NHS Foundation Trust, Manchester, United Kingdom, and §Division of Molecular \\ Pathology, Department of Pathology, Tottori University Faculty of Medicine, Yonago 683-8503, Japan
}

\section{ABSTRACT}

Background Merkel cell carcinomas (MCCs), clinically aggressive neuroendocrine skin cancers, are divided into Merkel cell polyomavirus (MCPyV)-positive and -negative tumors, which show different clinicopathological features and may develop through different mechanisms of carcinogenesis. Aberrant expression of activation-induced cytidine deaminase (AID) as a genomic modulator was demonstrated through pathogen-related NF- $x \mathrm{~B}$ signal in Helicobacter pylori-associated gastric cancer, adult T cell leukemia/lymphoma (HTLV-1), hepatoma (HCV), and Burkitt lymphoma (EBV).

Methods To elucidate the relation of aberrant AID expression in MCPyV-positive and -negative MCCs, we evaluated immunohistochemical expressions of AID and AID-regulating factors between $24 \mathrm{MCPyV}$-positive and 17 MCPyV-negative MCCs.

Results AID expression was significantly higher in $\mathrm{MCPyV}$-negative MCCs than MCPyV-positive ones $(P=0.026)$, although expression of NF- $x \mathrm{~B}$ p65 (phospho S536) (AID-enhancer) was significantly higher in MCPyV-positive MCCs than MCPyV-negative ones $(P=0.034)$. Expressions of PAX5 and c-Myb were not significantly different between these subgroups. Expressions of AID and AID-regulating factors were not correlated to prognosis of MCC patients.

Conclusion Our findings suggest that although pathogen-induced AID expression through upregulation of NF- $x \mathrm{~B}$ may be relevant to carcinogenesis of $\mathrm{MCPyV}$-positive MCCs, the significantly higher aberrant AID expression in MCPyV-negative MCCs is consistent with the fact that MCPyV-negative MCCs have an ex-

\footnotetext{
Corresponding author: Michiko Matsushita, $\mathrm{PhD}$

m-matsushita@med.tottori-u.ac.jp

Received 2017 June 9

Accepted 2017 July 3

Abbreviations: AID, activation-induced cytidine deaminase; CSR, class-switch recombination; DSS, disease-specific survival; HR, hazard ratio; MCC, Merkel cell carcinomas; MCPyV, Merkel cell polyomavirus; NF- $\varkappa \mathrm{B}$, Nuclear factor- $\varkappa \mathrm{B}$; OS, overall survival; PAX5, paired box gene 5; Rb, retinoblastoma; SHM, somatic hypermutation; UV, ultraviolet light
}

tremely higher mutation burden than MCPyV-positive ones.

Key words activation-induced cytidine deaminase; Merkel cell carcinoma; Merkel cell polyomavirus

Merkel cell carcinoma (MCC) is a clinically aggressive neuroendocrine skin cancer and Merkel cell polyomavirus $(\mathrm{MCPyV})$ is monoclonally integrated into the genome of approximately $80 \%$ of MCCs. ${ }^{1}$ Recently, we demonstrated clinical and pathogenetic differences between MCPyV-positive MCCs and MCPyV-negative MCCs; MCPyV-positive MCCs have a more round and narrow shape than MCPyV- negative MCCs, ${ }^{2}$ and MCPyV-positive MCC showed a better prognosis than MCPyV-negative MCCs. ${ }^{3-5}$ However, it has not been fully elucidated how the tumorigenic mechanism or pathway is different between MCPyV-positive and -negative MCCs.

Activation-induced cytidine deaminase (AID), a nucleotide-editing enzyme, is essential for the somatic hypermutation (SHM) and class-switch recombination (CSR) of the immunoglobulin gene ${ }^{6}$ and evidence of AID's involvement in carcinogenesis has been accumulated in not only B-cell lymphoma but non-B cell malignancy ${ }^{6,7}$ AID is a APOBEC family protein and induces off-target deamination of cytosine to uracil in DNA, and this AID-induced mutagenic U:G mismatch is considered as a common mutagenic mechanism in carcinogenesis. ${ }^{8}$ AID is also important as a genomic modulator in inflammation-associated cancer development in digestive organs including Helicobacter pylori $(H$. pylori)-associated gastric cancer, hepatitis $\mathrm{C}$ virus-positive hepatocellular carcinoma, colitis-associated colon cancers $^{9}$ and pancreatic cancer. ${ }^{10}$ Nuclear factor- $x \mathrm{~B}$ (NF$\varkappa \mathrm{B})$ activation in epithelial cells and malignant cells is involved in generating genomic instability through aberrant AID expression, cell growth, proliferation, survival, angiogenesis, and epithelial-to-mesenchymal transition (EMT). ${ }^{11}$ Transcription and expression of $A I D$ 
gene are controlled by many elements and transcription factors including NF- $x$ B, Stat6, C/EBP, Smad3/4, Myb, Pax5, E2A, E2f and BATF, bind to the AID regulatory regions. ${ }^{12}$ The expression and activity of AID are tightly regulated at the levels of transcription, post-transcription, and enzymatic function. Four distinct DNA regions (region I to IV) of the AID gene locus contain binding sites for multiple transcription factors. Region I functions as a promoter containing the binding site for NF$\mathrm{kB}$, a transcriptional activator. In B and non-B cells, enhancer elements in region II bind to the enhancer proteins PAX5 (paired box gene 5) and E2A, while silencer elements in region II bind to the silencer proteins $\mathrm{c}-\mathrm{Myb}$ and E2f in order to counter the activities of transcriptional enhancers. ${ }^{12}$ Honjo et al. described pathogen-induced AID expression in gastric cancer $(H$.pylori), adult T cell leukemia/lymphoma (ATLL) (HTLV-1), hepatoma (HCV), and Burkitt lymphoma (EBV), but not in classical Hodgkin's lymphoma (EBV). ${ }^{6}$ However, aberrant AID expression in MCPyV-associated $\mathrm{MCC}$ has not yet been elucidated. MCPyV is an oncogenic agent in a set of MCCs. ${ }^{3}$ In this study, we evaluated and compared the expression of AID and AID-regulating factors such as NF- $x \mathrm{~B}$ (enhancer), PAX5 (enhancer), and c-Myb (silencer) between MCPyV-positive and -negative MCCs in order to elucidate the association of MCPyV infection with expressions of AID and AID-regulating factors in the molecular pathogenesis of MCCs and clarify the question whether or not MCPyV-induced AID expression is a causative factor of carcinogenesis in $\mathrm{MCPyV}$-associated MCC.

\section{MATERIALS AND METHODS Samples}

This study was approved by the Institutional Review Board of the Faculty of Medicine at Tottori University (No.1216). We used $24 \mathrm{MCPyV}$-positive and 17 MCPyV-negative formalin-fixed paraffin- embedded (FFPE) MCC samples.

\section{MCPyV detection}

To detect MCPyV infection, real-time quantitative polymerase chain reaction (q-PCR) and immunohistochemistry with antibody to MCPyV-Large T protein (CM2B4) were performed according to our previous studies. ${ }^{5,13}$

\section{Immunohistochemistry}

Immunohistochemistry staining was performed on 4- $\mu \mathrm{m}$-thick paraffin sections as described previously. ${ }^{14}$ Primary antibodies were used with the following dilutions: mouse monoclonal anti-AID (clone ZA001, dilution 1/200; Thermo Fisher Scientific, Waltham,
MA), rabbit polyclonal anti-NF- $\varkappa$ B p65 (phospho S536) (1/500; Abcam, Cambridge, UK), rabbit monoclonal anti-c-Myb (clone EP769Y, dilution 1/150; LSBio, Seattle, WA), mouse monoclonal anti-PAX5 (clone DAK-Pax5, dilution 1/30; Agilent Technologies, Santa Clara, CA). Antigen retrieval of a rabbit polyclonal anti-NF- $x$ B p65 (phospho S536) was performed by incubating the sections with target retrieval solution ( $\mathrm{pH} 9.0$ ) for 20 minutes, other antibodies were performed by incubating the sections with citrate buffer ( $\mathrm{pH}$ 6.0) for 10 minutes using pressure cooker, then the immune complex was detected with an anti-mouse or anti-rabbit EnVision detection system and 3, 3'-diaminobenzidine and chromogenic substrate (DAKO).

For evaluating immunohistochemistry, H-score was used as described previously. ${ }^{15}$

The $\mathrm{H}$-score is performed as the sum of the percentage of staining multiplied by an ordinal value corresponding to the intensity level $(0=$ none, $1=$ weak, $2=$ moderate, $3=$ strong). The resulting score ranged from 0 (no staining in the tumor) to 300 (100\% diffuse strong staining of the tumor).

\section{Statistical analysis}

Immunohistochemical findings were analyzed in relation to MCPyV status using the Mann-Whitney $U$ test. To identify baseline patient and AID, NF- $x \mathrm{~B}$ p65 (phospho S536), c-Myb and PAX5 associated with prognosis, univariate hazard ratios were calculated with 95\% confidence intervals (CIs) using the Cox proportional-hazards model. The hazard ratio (HR) and 95\% $\mathrm{CI}$ were estimated using Cox hazard regression analysis for overall survival (OS), disease-specific survival (DSS) and stratification for MCPyV status, AID, NF- $\varkappa$ B p65(phospho S536), c-Myb and PAX5. The goodness of fit of each Cox model was evaluated using the likelihood ratio test, and the association between the individual variables and outcome was assessed using the forward Wald test selection $(P \leq 0.05$ was selected for entry into the model, and $P>0.1$ was selected for removal).

\section{RESULTS MCPyV detection}

MCPyV infection in MCCs was confirmed by the presence of MCPyV-DNA with q-PCR and positive staining of MCPyV-Large T antigen (CM2B4) in tumor cells. And the clinicopathological characteristics of 24 MCPyV-positive and 17 MCPyV-negative MCC cases are summarized in Table 1.

\section{Immunohistochemical staining}

The summary of immunohistochemical H-score for 
Table 1. Clinical information of the patients in this study

\begin{tabular}{|c|c|c|c|c|c|c|c|c|c|}
\hline Sample No. & $\begin{array}{l}\text { MCPyV } \\
\text { (q-PCR) }\end{array}$ & $\begin{array}{l}\text { Age } \\
\text { (years old) }\end{array}$ & Sex & $\begin{array}{l}\text { Tumor } \\
\text { size }(\mathrm{cm})\end{array}$ & $\begin{array}{l}\text { Initial } \\
\text { treatment }\end{array}$ & Organ & Site & Diagnosis & Month \\
\hline UK-M-1 & - & 81 & $\mathrm{~F}$ & 4 & $\mathrm{RE}$ & Dermis & Rt. shin & Combined MCC \& BD & 7 \\
\hline UK-M-2 & - & 81 & $\mathrm{~F}$ & $3.4 \times 3 \times 1.2$ & RE & Dermis & Rt. leg & Combined MCC \& BD & 44 \\
\hline UK-M-3 & - & 85 & $\mathrm{~F}$ & 2.1 & $\mathrm{RE}$ & Dermis & Lower leg & Combined MCC \& SqCC & 21 \\
\hline UK-M-4 & - & 93 & $\mathrm{~F}$ & 5 & $\mathrm{RE}$ & Dermis & Lt. cheek & Combined MCC \& SqCC & 6 \\
\hline UK-M-8 & - & 87 & $\mathrm{~F}$ & 1.4 & $\mathrm{RE}$ & Dermis & Forehead & Pure MCC & 12 \\
\hline UK-M-10 & - & 94 & $\mathrm{~F}$ & $6.5 \times 5 \times 2.5$ & PE & Dermis & $\begin{array}{l}\text { Rt. lateral leg with multiple } \\
\text { metastasis in the resion }\end{array}$ & Pure MCC & 16 \\
\hline UK-M-13 & - & 61 & M & $1.8 \times 1.5$ & RE & Dermis & Rt. shin & Pure MCC & 23 \\
\hline UK-M-14 & - & 86 & $\mathrm{~F}$ & $1.5 \times 1.5$ & $\mathrm{RE}$ & Dermis & Lt. dorsam foot & Combined MCC \& BD & 11 \\
\hline UK-M-20 & - & 82 & M & 3.5 & RE, RD & Dermis & Rt. knee & Combined MCC \& BD & 31 \\
\hline UK-M-5 & - & 81 & $\mathrm{~F}$ & 3.5 & $\mathrm{PE}$ & Dermis & Lt. leg with multiple satellites & Combined MCC \& SqCC & 18 \\
\hline UK-M-23 (meta) & - & & & & & $\mathrm{LN}$ & Lt. groin & & \\
\hline UK-M-17 & - & 83 & $\mathrm{~F}$ & $1.7 \times 1.4$ & PE & Dermis & Lt. lower eyelid & Combined MCC \& BCC & 39 \\
\hline UK-M-17 (meta) & - & & & & & Dermis & Conjunctiva & & \\
\hline UK-M-18 & - & 94 & $\mathrm{~F}$ & $5 \times 4 \times 2$ & $\mathrm{RD}, \mathrm{RE}$ & Dermis & Rt. temple & Pure MCC & 6 \\
\hline UK-M-31 (meta) & - & & & & & $\mathrm{LN}$ & Rt. parotid & & \\
\hline MCC50 & - & 82 & M & 1.5 & $\mathrm{RE}$ & Dermis & Dorsum of the hand & Combined MCC \& BD & 13 \\
\hline MCC51 (meta) & - & & & & & $\mathrm{LN}$ & Axilla & & \\
\hline UK-M-7 & + & 68 & M & $2.7 \times 1.7 \times 0.8$ & RE & Dermis & Rt. knee & Pure MCC & 59 \\
\hline UK-M-9 & + & 69 & M & $5 \times 5 \times 4$ & $\mathrm{RE}$ & Dermis & Rt. groin & Pure MCC & 55 \\
\hline UK-M-11 & + & 61 & $\mathrm{~F}$ & 1.2 & $\mathrm{RE}$ & Dermis & Rt. cheek & Pure MCC & 18 \\
\hline UK-M-16 & + & 63 & $\mathrm{~F}$ & $2.5 \times 2.5$ & RE, RD & Dermis & Lt. upper arm & Pure MCC & 70 \\
\hline UK-M-19 & + & 85 & $\mathrm{~F}$ & $4 \times 2.8 \times 1.3$ & $\mathrm{RE}$ & Dermis & Rt. forearm & Pure MCC & 21 \\
\hline UK-M-22 & + & 74 & $\mathrm{~F}$ & $6 \times 5.5 \times 2$ & RE & Dermis & Lt. elbow & Pure MCC & 72 \\
\hline MCC36 & + & 87 & $\mathrm{~F}$ & $2 \times 2.3$ & RE & Dermis & Nasal ala & Pure MCC & 3 \\
\hline MCC37 & + & 73 & $\mathrm{~F}$ & 0.9 & $\mathrm{RE}$ & Dermis & Cheek & Pure MCC & 29 \\
\hline MCC38 & + & 83 & $\mathrm{~F}$ & $1.1 \times 1.0 \times 0.8$ & $\mathrm{RE}$ & Dermis & Cheek & Pure MCC & 2 \\
\hline MCC45 & + & 64 & $\mathrm{~F}$ & $2 \times 3$ & Unknown & Dermis & Lower thigh & Pure MCC & Unknown \\
\hline MCC46 & + & 67 & $\mathrm{~F}$ & $2 \times 1.5$ & Unknown & Hypodermis & Buttock & Pure MCC & Unknown \\
\hline MCC47 & + & 90 & M & 2 & Unknown & Dermis & Humerus & Pure MCC & Unknown \\
\hline MCC53 & + & 61 & $\mathrm{~F}$ & 1.9 & $\mathrm{RE}$ & Dermis & Earflap & Pure MCC & 28 \\
\hline MCC55 & + & 75 & M & $1.9 \times 1.7$ & Unknown & Dermis & Cheek & Pure MCC & Unknown \\
\hline MCC57 & + & 77 & $\mathrm{~F}$ & Hen's egg size & CT, RE & Hypodermis & Knee & Pure MCC & 53 \\
\hline MCC61 & + & 59 & $\mathrm{~F}$ & $1.4 \times 1.1$ & RE & Dermis & Lower thigh & Pure MCC & 35 \\
\hline MCC66 & + & 86 & $\mathrm{~F}$ & 10 & $\mathrm{RD}$ & Dermis & Superior eyelid & Pure MCC & 1 \\
\hline MCC70 & + & NA & M & 3 & $\mathrm{RE}$ & Dermis & Thigh & Pure MCC & 34 \\
\hline MCC73 & + & 90 & $\mathrm{~F}$ & 2 & $\mathrm{RD}$ & Dermis & Cheek & Pure MCC & 3 \\
\hline MCC83 & + & 71 & $\mathrm{~F}$ & 2 & RE, RD & Dermis & Lt. thigh & Pure MCC & 31 \\
\hline UK-M-21 & + & 46 & $\mathrm{~F}$ & $3 \times 2.5$ & $\mathrm{RE}$ & Dermis & Lt. buttock & Pure MCC & 59 \\
\hline UK-M-21 (meta) & + & & & & & Dermis & & & \\
\hline MCC32 & + & 66 & M & $2 \times 2.5$ & $\mathrm{RE}$ & Dermis & Thigh & Pure MCC & 2 \\
\hline MCC33 (meta) & + & & & & & LN & Inguina & & \\
\hline
\end{tabular}

BD, Bowen's disease; CT, chemotherapy; F, female; LN, lymph node; Lt., left; M, male; MCC, Merkel cell carcinoma; MCPyV, Merkel cell polyomavirus; meta, metastasis; NA, not available; PE, palliative excision; q-PCR, quantitative polymerase chain reaction; RD, radiation; RE, radical excision; Rt., right; SqCC, squamous cell carcinoma.

AID, NF- $x$ B p65 (phospho S536), PAX5 and c-Myb, and the representative immunostainings in the MCC subgroups are shown in Table 2, Figure 1, respectively. NF- $x$ B p65 (phospho S536) was expressed only in the nuclei.

AID expression was significantly lower in
MCPyV-positive MCCs than MCPyV-negative ones $(P=0.026)$, although expression of NF- $x \mathrm{~B}$ p65 (phospho S536) (AID-enhancer) was significantly higher in MCPyV-positive MCCs than MCPyV-negative ones $(P=$ 0.034) (Table 3, Fig.2). There were no statistically significant differences between MCPyV-positivity in MCCs 
and the other AID-regulating factors (PAX5 and c-Myb) (Table 3, Fig.2).

In addition, there was a statistically significant association between AID and NF- $x \mathrm{~B}$ p65 (phospho S536) expressions $(P<0.05)$ but no statistically significant correlations between AID and other AID-regulating factors (PAX5 or c-Myb) in MCCs.

Table 2. Summary of immunohistochemical H-scores for AID and AID-regulating factors in MCPyV-positive and -negative MCCs

\begin{tabular}{|c|c|c|c|c|c|c|}
\hline \multirow[b]{2}{*}{ Sample No. } & \multirow{2}{*}{$\frac{\mathrm{q}-\mathrm{PCR}}{M C P y V-\mathrm{DNA}}$} & \multirow{2}{*}{ 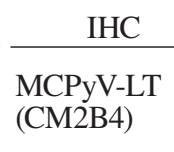 } & \multicolumn{4}{|c|}{ IHC (H-score) } \\
\hline & & & AID & $\begin{array}{l}\text { NF- } x \text { B p65 } \\
\text { (phospho S536) } \\
\text { (AID-enhancer) }\end{array}$ & $\begin{array}{l}\text { PAX5 } \\
\text { (AID-enhancer) }\end{array}$ & $\begin{array}{l}\text { c-Myb } \\
\text { (AID-silencer) }\end{array}$ \\
\hline UK-M-1 & - & - & 180 & 0 & 150 & 200 \\
\hline UK-M-2 & - & - & 225 & 0 & 125 & 84 \\
\hline UK-M-3 & - & - & 245 & 0 & 200 & 165 \\
\hline UK-M-4 & - & - & 180 & 15 & 150 & 140 \\
\hline UK-M-8 & - & - & 150 & 20 & 170 & 235 \\
\hline UK-M-10 & - & - & 205 & 120 & 142 & 190 \\
\hline UK-M-13 & - & - & 220 & 100 & 100 & 250 \\
\hline UK-M-14 & - & - & 185 & 200 & 100 & 270 \\
\hline UK-M-20 & - & - & 185 & 0 & 190 & 175 \\
\hline UK-M-5 & - & - & 90 & 10 & 170 & 110 \\
\hline UK-M-23 (meta) & - & - & 190 & 39 & 150 & 265 \\
\hline UK-M-17 & - & - & 150 & 10 & 160 & 140 \\
\hline UK-M-17 (meta) & - & - & 300 & 20 & 190 & 235 \\
\hline UK-M-18 & - & - & 180 & 15 & 56 & 130 \\
\hline UK-M-31 (meta) & - & - & 100 & 90 & 205 & 120 \\
\hline MCC50 & - & - & 195 & 20 & 85 & 170 \\
\hline MCC51 (meta) & - & - & 228 & 35 & 173 & 80 \\
\hline UK-M-7 & + & + & 175 & 40 & 155 & 260 \\
\hline UK-M-9 & + & + & 145 & 31 & 140 & 210 \\
\hline UK-M-11 & + & - & 140 & 99 & 130 & 160 \\
\hline UK-M-16 & + & + & 190 & 85 & 205 & 190 \\
\hline UK-M-19 & + & + & 205 & 225 & 160 & 255 \\
\hline UK-M-22 & + & + & 150 & 60 & 180 & 200 \\
\hline MCC36 & + & + & 135 & 165 & 200 & 205 \\
\hline MCC37 & + & + & 240 & 53 & 105 & 225 \\
\hline MCC38 & + & + & 125 & 60 & 110 & 230 \\
\hline MCC45 & + & + & 165 & 66 & 105 & 205 \\
\hline MCC46 & + & + & 108 & 14 & 115 & 5 \\
\hline MCC47 & + & + & 165 & 40 & 65 & 103 \\
\hline MCC53 & + & + & 100 & 120 & 110 & 126 \\
\hline MCC55 & + & + & 80 & 393 & 105 & 260 \\
\hline MCC57 & + & + & 195 & 80 & 170 & 245 \\
\hline MCC61 & + & + & 170 & 130 & 200 & NA \\
\hline MCC66 & + & + & 70 & 40 & 200 & 245 \\
\hline MCC70 & + & + & 190 & 30 & 180 & 87 \\
\hline MCC73 & + & + & 215 & 240 & 240 & 93 \\
\hline MCC83 & + & + & 185 & 190 & 170 & 150 \\
\hline UK-M-21 & + & + & 250 & 10 & 90 & 150 \\
\hline UK-M-21 (meta) & + & + & 280 & 11 & 170 & 260 \\
\hline MCC32 & + & + & 120 & 30 & 180 & 130 \\
\hline MCC33 (meta) & + & + & 90 & 55 & 90 & 145 \\
\hline
\end{tabular}

AID, activation-induced cytidine deaminase; c-Myb, a member of MYV family; IHC, immunohistochemistry; LT, Large T antigen; MCC, Merkel cell carcinoma; MCPyV, Merkel cell polyomavirus; meta, metastasis; NA, not available; NE, not examined; NF- $\varkappa \mathrm{B}$, Nuclear factor kappa-light-chain-enhancer of activated B cells; PAX5, Paired Box 5; q-PCR, quantitative polymerase chain reaction. 
Table 3. Comparison of expressions of AID and AID-regulating factors in MCCs based on MCPyV status

\begin{tabular}{lccc}
\hline Clinicopathological data & MCPyV-positive MCCs & MCPyV-negative MCCs & $P$-value $\dagger$ \\
\hline Age (y.o.; mean \pm SD) & $74.6 \pm 9.8$ & $83.3 \pm 9.1$ & 0.065 \\
Sex (male/female) & $6 / 16$ & $3 / 10$ & 0.56 \\
Stage (I/ II/ III) & $9 / 11 / 2$ & $2 / 7 / 2$ & 0.835 \\
AID H-score (mean \pm SD) & $157.4 \pm 44.0$ & $197.2 \pm 27.4$ & $0.026^{*}$ \\
NF- $x$ B p65 (phospho S536) H-score (mean \pm SD) & $108.1 \pm 91.8$ & $50.6 \pm 68.3$ & $0.034^{*}$ \\
PAX5 H-score (mean \pm SD) & $152.3 \pm 44.4$ & $147.4 \pm 33.5$ & 0.627 \\
c-Myb H-score (mean \pm SD) & $181.8 \pm 69.2$ & $189.9 \pm 54.5$ & 1 \\
\hline
\end{tabular}

*Statistically significant. †Mann-Whitney $U$ test.

AID, activation-induced cytidine deaminase; c-Myb, a member of MYV family; MCC, Merkel cell carcinoma; MCPyV, Merkel cell polyomavirus; NF- ^B, Nuclear factor kappa-light-chain-enhancer of activated B cells; PAX5, Paired Box 5; y.o., years old.

MCPyV-positive case

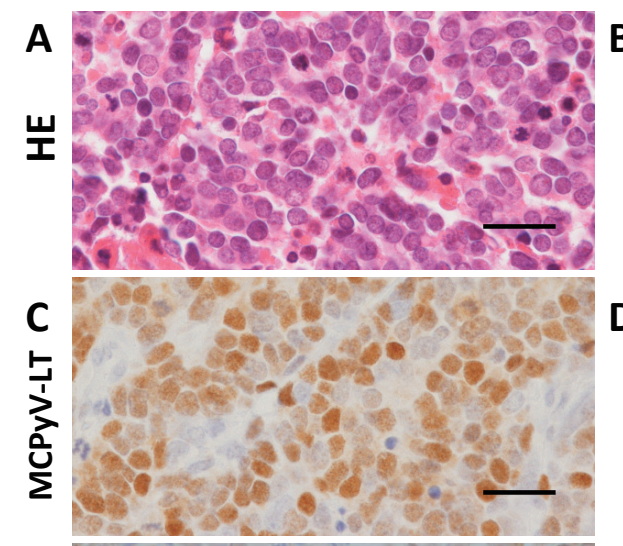

\section{E}
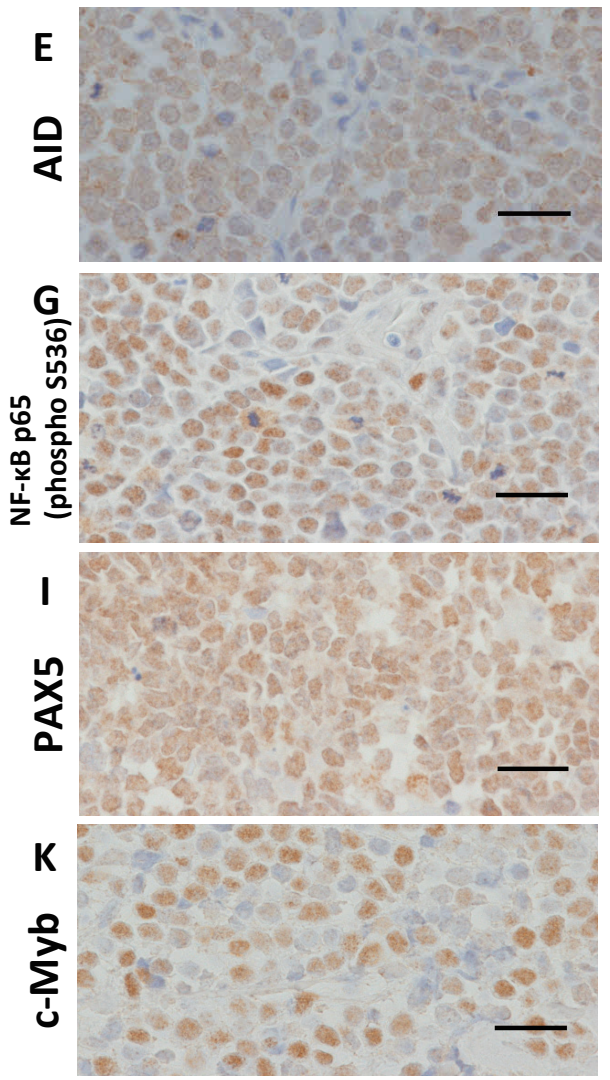

\section{MCPyV-negative case}

B

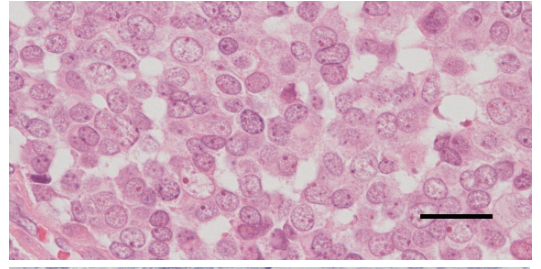

D

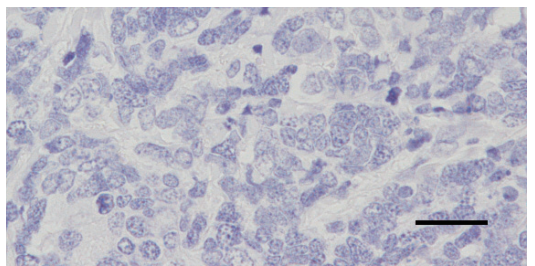

$\mathrm{F}$

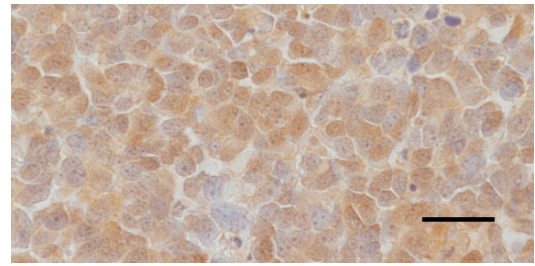

H

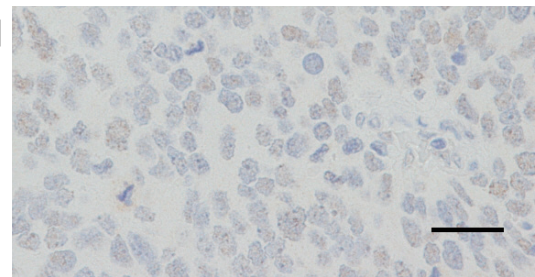

J

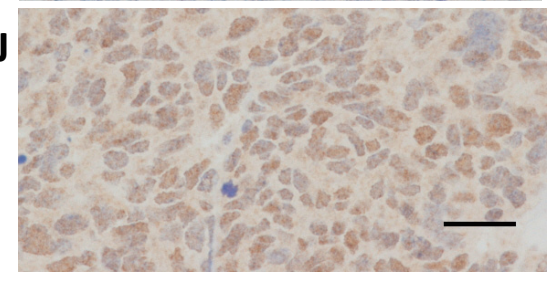

$\mathbf{L}$

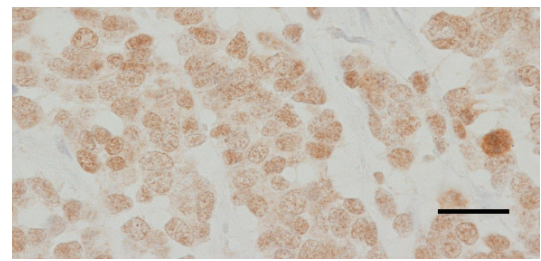

Fig. 1. Representative images of immunostaining of MCPyV-positive and MCPyV-negative MCCs. Representative images of IHC of AID and AID-regulating factors [NF- $x \mathrm{~B}$ p65 (phospho S536) (AID-enhancer), PAX5 (AID-enhancer), and c-Myb (AID-silencer)] in MCPyV-positive $\operatorname{MCCs}(\mathbf{A}, \mathbf{C}, \mathbf{E}, \mathbf{G}, \mathbf{I}$, and $\mathbf{K})$ and MCPyV-negative MCCs (B, D, F, H, $\mathbf{J}$, and $\mathbf{L}$ ) are shown. MCPyV-negative MCC tumor cells (B) had more irregular nuclear shapes and more abundant cytoplasm than did MCPyV-positive MCC cells (A). MCPyV DNA-positive MCCs showed strong positive nuclear immunoreactivity for MCPyV-LT (C), whereas MCPyV DNA-negative MCCs were negative for MCPyV-LT (D). AID expression was observed with significantly higher $\mathrm{H}$-score in MCPyV-negative MCCs (F) than in MCPyV-positive MCCs (E) (H-score, mean $\pm \mathrm{SD}$, $197.2 \pm 27.4$ versus $157.4 \pm 44.0 ; P=$ 0.026). NF-xB p65 (phospho S536) expression was significantly higher in MCPyV-positive MCCs $(\mathbf{G})$ than in MCPyV-negative MCCs $(\mathbf{H})(\mathrm{H}$ score: mean $\pm \mathrm{SD}, 108.1 \pm 91.8$ versus $50.6 \pm 68.3 ; P=0.034)$. However, no significant differences were observed in the H-scores of PAX5 and c-Myb between MCPyV-positive and -negative MCCs.

$\mathbf{A}$ and $\mathbf{B}$, hematoxylin and eosin stain; A through $\mathbf{L}$, IHC, scale bar $=25 \mu \mathrm{m}$. AID, activation-induced cytidine deaminase; c-Myb, a member of MYV family; HE, hematoxylin-eosin; LT, Large T antigen; MCC, Merkel cell carcinoma; MCPyV, Merkel cell polyomavirus; NF- xB, Nuclear factor kappa-light-chain-enhancer of activated B cells; PAX5, Paired Box 5. 


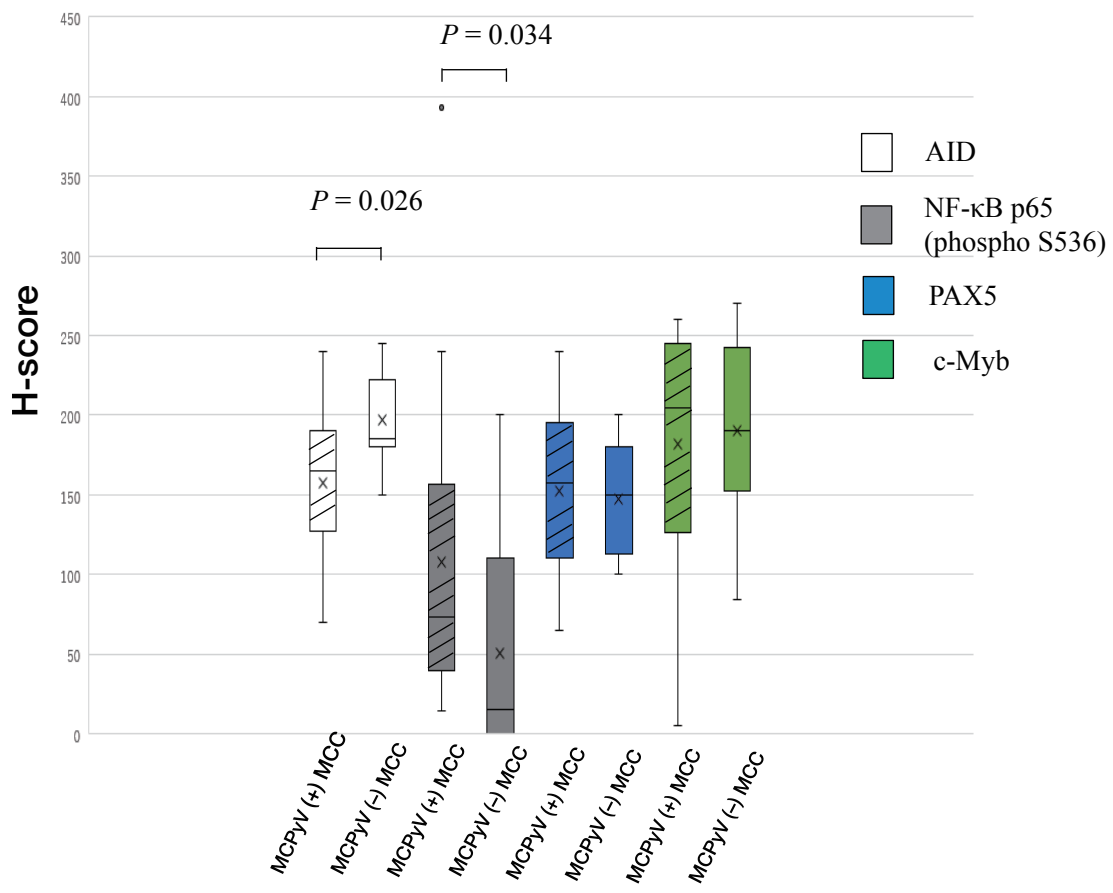

Fig. 2. Comparison of the immunohistochemical H-score for AID, NF- $x$ B p65 (phospho S536), PAX5 and c-Myb between MCPyV-positive and -negative MCCs. AID expression was significantly lower in MCPyV-positive MCCs than MCPyV-negative ones $(P=0.026)$ while NF- $x$ B p65 (phospho S536) expression was significantly higher in MCPyV-positive MCCs than MCPyV-negative ones $(P=0.034)$. No statistically significant difference was observed on $\mathrm{H}$-scores of PAX5 and c-Myb expression between MCPyV-positive and -negative MCCs. AID, activation-induced cytidine deaminase; c-Myb, a member of MYV family; MCC, Merkel cell carcinoma; MCPyV, Merkel cell polyomavirus; NF- $x$ B, Nuclear factor kappa-light-chain-enhancer of activated B cells; PAX5, Paired Box 5.

\section{Statistical analysis for survival}

Results of univariate and multivariate Cox regression analyses for all patients can be seen in Table 4. In univariate analysis, MCPyV-positivity was a favorable parameter associated with a significant increase of both OS (HR: 0.101, $P=0.001$ ) and DSS (HR: 0.090, $P=$ 0.003 ), while male gender was significantly unfavorable in DSS (HR: 0.090, $P=0.003$ ) but not in OS (HR: 2.399, $P=0.250$ ). With multivariate analysis, only the presence of MCPyV was found to be a significantly favorable prognostic factor for both OS (HR: $0.03, P<0.001$ ) and DSS (HR: 0.025, $P=0.006$ ), while male gender and advanced stage (stage II and III) were significantly unfavorable only for OS (HR: 10.89, $P=0.045$; HR: 14.39, $P$ $=0.026$, respectively). Expressions of AID and AID-regulating factors were not significantly associated with OS and DSS.

\section{DISCUSSION}

AID expression of Merkel cell carcinoma with lymphnode metastasis was reported in a case report. ${ }^{16}$ However, for the first time, we focused to study on the association of expression of AID, a genomic mutator, with pathogenetic differences between MCPyV-positive and negative MCCs and demonstrated that MCPyV-negative MCCs showed a significantly higher expression of AID than MCPyV-positive ones. This finding is consistent with the reported facts that MCPyV-negative MCCs displayed higher overall mutation burden than MCPyV-positive ones..$^{17,18}$
Our previous studies showed some clinicopathological differences between MCPyV-positive and -negative MCCs; MCPyV-positive MCCs showed a significantly higher expression of retinoblastoma protein and less p53 expression compared to MCPyV-negative MCCs, and frequency of TP53 non-ultraviolet signature mutation was significantly higher in MCPyV-negative MCCs than in MCPyV-positive MCCs. ${ }^{4}$ And we also reported that Akt phosphorylation at T308 in activation of the Akt/ mammalian target of rapamycin (mTOR)/4E-binding protein 1 (4E-BP1) signaling pathway, was significantly greater in $\mathrm{MCPyV}$-negative than in $\mathrm{MCPyV}$-positive $\mathrm{MCCs}^{19}$ and that lower expression of CADM1 and higher expression of MAL in MCCs are associated with MCPyV infection and better prognosis. ${ }^{15}$ It can be considered that $\mathrm{MCPyV}$-positive and $\mathrm{MCPyV}$-negative MCCs have different tumorigenic pathways; the integrated-mutated form of MCPyV is directly involved in "virus-mediated" tumorigenesis, whereas the accumulation of more complicating genetic aberrations is required for "nonviral" tumorigenesis; and both may develop under systemic/local impairment of host immune surveillance caused by UV irradiation, immunosenescence, use of immunosuppressants, and other factors. ${ }^{3}$

Cancer cells are considered to be generated from the stepwise accumulation of genetic alterations in various genes in inflammation-associated carcinogenesis. ${ }^{9}$ AID, a nucleotide-editing enzyme that is essential for SHM and CSR of the immunoglobulin gene, is also known to play a role as a genomic mutator in carcinogenesis. ${ }^{11}$ 
Table 4. Univariate and multivariate cox proportional hazard analysis for mortality in MCC cases

\begin{tabular}{|c|c|c|c|c|c|c|}
\hline \multirow{2}{*}{$\begin{array}{l}\text { Factors } \\
\text { Univariate analysis }\end{array}$} & \multicolumn{3}{|c|}{ Overall survival } & \multicolumn{3}{|c|}{ MCC specific survival } \\
\hline & HR & $95 \% \mathrm{CI}$ & $P$-value & HR & $95 \% \mathrm{CI}$ & $P$-value \\
\hline Age $(>75 / \leq 75$ y.o. $)$ & 4.250 & $0.559-32.312$ & 0.162 & 2.919 & $0.371-22.970$ & 0.309 \\
\hline Sex (male/female) & 2.399 & $0.540-19.664$ & 0.250 & 0.090 & $0.180-0.441$ & 0.003 \\
\hline Stage (stageII, III/stageI) & 2.106 & $0.462-9.595$ & 0.336 & 1.692 & $0.205-13.957$ & 0.625 \\
\hline MCPyV-positive/-negative & 0.101 & $0.028-0.370$ & 0.001 & 0.090 & $0.018-0.441$ & 0.003 \\
\hline AID H-score $(>160$ vs $\leq 160)$ & 1.174 & $0.400-3.444$ & 0.770 & 0.993 & $0.283-3.491$ & 0.992 \\
\hline NF- $x$ B H-score $(>65$ vs $\leq 65)$ & 0.834 & $0.287-2.423$ & 0.739 & 0.718 & $0.188-2.742$ & 0.628 \\
\hline PAX5 H-score (> 130 vs $\leq 130)$ & 1.448 & $0.465-4.512$ & 0.523 & 2.147 & $0.462-9.985$ & 0.330 \\
\hline c-Myb H-score (> 160 vs $\leq 160)$ & 1.392 & $0.504-3.848$ & 0.524 & 1.051 & $0.319-3.463$ & 0.935 \\
\hline Factors & \multicolumn{3}{|c|}{ Overall survival } & \multicolumn{3}{|c|}{ MCC specific survival } \\
\hline Multivariate analysis & HR & $95 \% \mathrm{CI}$ & $P$-value & HR & $95 \% \mathrm{CI}$ & $P$-value \\
\hline Age $(>75 / \leq 75$ y.o. $)$ & 0.23 & $0.010-5.166$ & 0.356 & 0.064 & $0.001-2.866$ & 0.156 \\
\hline Sex (male/female) & 10.89 & $1.051-112.832$ & 0.045 & 2.665 & $0.252-28.180$ & 0.415 \\
\hline Stage (stageII,III/stageI) & 14.39 & $1.369-151.338$ & 0.026 & 6.632 & $0.197-222.796$ & 0.291 \\
\hline MCPyV-positive/-negative & 0.03 & $0.004-0.207$ & $<0.001$ & 0.025 & $0.002-0.346$ & 0.006 \\
\hline AID H-score $(>160$ vs $\leq 160)$ & 1.04 & $0.208-5.162$ & 0.966 & 2.467 & $0.273-22.286$ & 0.421 \\
\hline $\begin{array}{l}\text { NF- } x \text { B p } 65 \text { (phospho S536)H-score } \\
(>65 \text { vs } \leq 65)\end{array}$ & 1.43 & $0.324-6.315$ & 0.636 & 1.401 & $0.200-9.798$ & 0.734 \\
\hline PAX5 H-score (> 130 vs $\leq 130)$ & 2.40 & $0.426-13.536$ & 0.321 & 18.006 & $0.938-345.730$ & 0.055 \\
\hline c-Myb H-score (> 160 vs $\leq 160)$ & 4.43 & $0.697-28.102$ & 0.115 & 0.846 & $0.078-9.209$ & 0.891 \\
\hline
\end{tabular}

AID, activation-induced cytidine deaminase; CI, confidence interval; c-Myb, a member of MYV family; HR, hazard ratio; MCC, Merkel cell carcinoma; MCPyV, Merkel cell polyomavirus; NF- $\varkappa$ B, Nuclear factor kappa-light-chain-enhancer of activated B cells; PAX5, Paired Box 5; y.o., years old.

Pathogenic bacterial or viral factors and subsequent inflammatory reactions in $H$. pylori-related gastritis, chronic viral hepatitis, Barrett's esophagus, and inflammatory bowel disease lead to the aberrant expression of AID in various epithelial cells via NF- $x$ B activation, which causes the accumulation of genetic alterations in tumor-related genes. ${ }^{11}$

Most MCCs are MCPyV-infected tumor. Our study revealed that the aberrant expression of AID was significantly lower in MCPyV-positive MCCs than in MCPyV-negative MCCs $(P=0.026)$, although aberrant NF- $x$ B p65 (phospho S536) expression (an AID-enhancer) was significantly higher in MCPyV-positive MCCs than in MCPyV-negative MCCs $(P=0.034)$. Aberrant expression of PAX5 (an AID-enhancer) and c-Myb (an AID-silencer) was not significantly different between MCPyV-positive and -negative MCCs, and also that MCPyV was likely to be involved in pathogen-induced AID expression via NF- $x \mathrm{~B}$ activation in MCPyV-positive MCCs. These results suggest that the genomic modulation of tumor-related genes by $\mathrm{MCPyV}$-induced AID expression is a causative mechanism in the carcinogenesis of MCPyV-positive MCCs. However, Unexpectedly, the aberrant expression of AID was higher in MCPyV-negative MCCs with lower NF$\varkappa \mathrm{B}$ p65 (phospho S536) expression than in MCPyV-positive MCCs with higher NF- $x$ B p65 (phospho S536) expression $(P=0.026)$. This finding indicates that additional factors may modulate expression level of AID; the other enhancers and/or silencers of AID may influence the expression of AID in MCCs. Recently, in fact, it is known that not only NF- $\varkappa \mathrm{B}$, but also Homeobox protein $\mathrm{C} 4$ may promote the transcription of AID and E2f, a transcription factor targeted by $\mathrm{Rb}$ protein, is also a silencer for AID. ${ }^{12}$

Cimino PJ et $\mathrm{al}^{20}$ performed whole exome sequencing on five MCPyV-positive cases and three MCPyV-negative cases and the retinoblastoma gene $(R B 1)$ was found to have nonsense truncating gene mutations in all three MCPyV-negative cases, whereas no such mutations were found in the MCPyV-positive cases. While MCPyV-positive MCCs is believed to undergo retinoblastoma dysregulation through viral large $\mathrm{T}$ antigen expression, their findings demonstrate that somatic mutations of $R B 1$ in MCPyV-negative MCCs lead to retinoblastoma dysregulation through an alternative pathway. Harms PW et al performed integrative sequencing on 16 cases of MCC and reported 
that MCPyV-negative tumors displayed high overall mutation burden $(10.09 \pm 2.32$ mutations $/ \mathrm{Mb})$ and were characterized by a prominent $\mathrm{UV}$-signature pattern with $\mathrm{C}>\mathrm{T}$ transitions comprising $85 \%$ of mutations, while mutation burden was low in MCPyV-positive tumors $(0.40 \pm 0.09$ mutations/ $\mathrm{Mb})$ and lacked a UV signature. Recent exome sequencing studies on $49 \mathrm{MCCs}^{18}$ also reconfirmed the dramatical differences of gene mutations between $\mathrm{MCPyV}$-positive and -negative MCCs; MCPyV-negative MCCs have a high mutation burden (median of 1121 somatic single nucleotide variants (SSNVs) per-exome) with frequent mutations in $R B I$ and TP53 and additional damaging mutations in genes in the chromatin modification ( $A S X L 1, M L L 2$, and $M L L 3)$, JNK (MAP3K1 and TRAF7), and DNA-damage pathways (ATM, MSH2, and BRCA1). In contrast, MCPyV-positive MCCs harbor few SSNVs (median of 12.5 SSNVs/tumor) with none in the genes listed above. Goh et al. ${ }^{18}$ also reconfirmed that MCPyV-negative MCCs were significantly enriched for $\mathrm{C}>\mathrm{T}$ transitions (median of $86 \%$ of SSNVs in MCPyV-negative MCCs vs. $47 \%$ of SSNVs in MCPyV-positive MCCs; $P=3.1 \mathrm{E}$ 7; two-sided Mann-Whitney test) and hypothesized that the enrichment of $\mathrm{C}>\mathrm{T}$ transitions were a result of ultraviolet light (UV), because MCCs develop on the skin. However, they also pointed that $\mathrm{C}>\mathrm{T}$ transitions can be caused by other mechanisms, e.g. such as aging and impaired mismatch repair ${ }^{21}$ and reported that a median of $66 \%$ of SSNVs per MCPyV-negative MCC could be attributed to UV exposure.

Recent high-throughput sequencing of large numbers of human cancer genomes showed that mutations at cytosine residues, particularly $\mathrm{C}$ to $\mathrm{T}$ transitions, are the most prevalent mutations in human cancer, highlighting enzymatic deamination of cytosine to uracil like AID-induced U:G mismatches in DNA as a potential source of mutagenesis. ${ }^{21}$ AID-induced U:G mismatches from $\mathrm{C}: \mathrm{G}$ in DNA are partially transited by replication over deoxyuridines to T:A, resulting in AID-induced $\mathrm{C}>\mathrm{T}$ transition (C:G to T:A transition). Despite AID's important physiological functions for SHM and CSR in Ig gene, these host defense mechanisms entail a high risk of potentially carcinogenic off-target genomic mutagenesis. Our finding of the significantly higher aberrant AID expression in MCPyV-negative MCCs than in MCPyV-positive MCCs $(P=0.026)$ is, compatible with the reported exome sequencing data ${ }^{17,18}$ that MCPyV-negative MCCs have an extremely higher mutation burden than MCPyV-positive ones, although UV looks like a major mutagenic factor in $\mathrm{MCPyV}$-negative MCCs.

In conclusion, lower expression of AID and higher expression of NF- $x \mathrm{~B}$ p65 (phospho S536) were significantly associated with MCPyV-positive MCCs. While pathogen (MCPyV)-induced AID expression through upregulation of NF- $x \mathrm{~B}$ may be relevant to carcinogenesis of MCPyV-positive MCCs, the other regulating factors for AID may influence the higher AID expression in MCPyV-negative MCCs. The higher aberrant expression of AID, a mutagenic enzyme, and more frequent AID-related mutation signatures observed in MCPyV-negative MCCs associated with poor prognosis in this study is compatible with the fact that MCPyV-negative MCCs have an extremely higher mutation burden than MCPyV-positive ones.

Acknowledgments: This work was supported by JSPS KAKENHI Grant Numbers JP 26460433 and 26860238.

The authors declare no conflict of interest.

\section{REFERENCES}

1 Feng H, Shuda M, Chang Y, Moore PS. Clonal integration of a polyomavirus in human Merkel cell carcinoma. Science. 2008;319:1096-100. PMID: 18202256

2 Kuwamoto S, Higaki H, Kanai K, Iwasaki T, Sano H, Nagata $\mathrm{K}$, et al. Association of Merkel cell polyomavirus infection with morphologic differences in Merkel cell carcinoma. Hum Pathol. 2011;42:632-40. PMID: 21277612.

3 Kuwamoto S. Recent advances in the biology of Merkel cell carcinoma. Hum Pathol. 2011;42:1063-77. PMID: 21641014.

4 Higaki-Mori H, Kuwamoto S, Iwasaki T, Kato M, Murakami I, Nagata K, et al. Association of Merkel cell polyomavirus infection with clinicopathological differences in Merkel cell carcinoma. Hum Pathol. 2012;43:2282-91. PMID: 22795182.

5 Iwasaki T, Matsushita M, Kuwamoto S, Kato M, Murakami I, Higaki-mori $\mathrm{H}$, et al. Usefulness of significant morphologic characteristics in distinguishing between Merkel cell polyomavirus-positive and Merkel cell polyomavirus-negative Merkel cell carcinomas. Hum Pathol. 2013;44:1912-7. PMID: 23664542

6 Honjo T, Kobayashi M, Begum N, Kotani A, Sabouri S, Nagaoka H. The AID dilemma: Infection or cancer? Advances in Cancer Research. 2012;113:1-44. PMID: 22429851.

7 Rebhandl S, Huemer M, Greil R, Geisberger R. AID/APOBEC deaminases and cancer. Oncoscience. 2015;2:320-33. PMID: 26097867.

8 Pettersen HS, Galashevskaya A, Doseth B, Sousa MM, Sarno A, Visnes T, et al. AID expression in B-cell lymphomas causes accumulation of genomic uracil and a distinct AID mutational signature. DNA Repair (Amst). 2015;25:60-71. PMID: 25486549

9 Chiba T, Marusawa H, Ushijima T. Inflammation-associated cancer development in digestive organs: mechanisms and roles for genetic and epigenetic modulation. Gastroenterology. 2012;143:550-63. PMID: 22796521.

10 Sawai Y, Kodama Y, Shimizu T, Ota Y, Maruno T, Eso Y, et al. Activation-Induced Cytidine Deaminase Contributes to Pancreatic Tumorigenesis by Inducing Tumor-Related Gene Mutations. Cancer Res. 2015;75:3292-301. PMID: 26113087.

11 Shimizu T, Marusawa H, Endo Y, Chiba T. Inflammation-me- 
diated genomic instability: roles of activation-induced cytidine deaminase in carcinogenesis. Cancer Sci. 2012;103:1201-6. PMID: 22469133.

12 Zan H, Casali P. Regulation of Aicda expression and AID activity. Autoimmunity. 2013;46:83-101. PMID: 23181381.

13 Matsushita M, Iwasaki T, Kuwamoto S, Kato M, Nagata K, Murakami I, et al. Merkel cell polyomavirus (MCPyV) strains in Japanese merkel cell carcinomas (MCC) are distinct from Caucasian type MCPyVs: genetic variability and phylogeny of MCPyV genomes obtained from Japanese MCPyV-infected MCCs. Virus Genes. 2014;48:233-42. PMID: 24353025.

14 Iwasaki T, Matsushita M, Nonaka D, Kato M, Nagata K, Murakami I, et al. Phosphohistone-H3 (PHH3) is prognostic relevant in Merkel cell carcinomas but Merkel cell polyomavirus is a more powerful prognostic factor than AJCC clinical stage, PHH3, Ki-67 or mitotic indices. Pathol Int. 2015;65:404-9. PMID: 25982855.

15 Iwasaki T, Matsushita M, Nonaka D, Nagata K, Kato M, Kuwamoto S, et al. Lower expression of CADM1 and higher expression of MAL in Merkel cell carcinomas are associated with Merkel cell polyomavirus infection and better prognosis. Hum Pathol. 2016;48:1-8. PMID: 26772392.

16 Watabe R, Nakamura M. Expression of activation-induced cytidine deaminase in Merkel cell carcinoma with lymph- node metastasis. Eur J Dermatol.2013;23:539-40. PMID: 24001680.

17 Harms PW, Vats P, Verhaegen ME, Robinson DR, Wu YM, Dhanasekaran SM, et al. The Distinctive Mutational Spectra of Polyomavirus-Negative Merkel Cell Carcinoma. Cancer Res. 2015;75:3720-7. PMID: 26238782.

18 Goh G, Walradt T, Markarov V, Blom A, Riaz N, Doumani R, et al: Mutational landscape of MCPyV-positive and MCPyV-negative Merkel cell carcinomas with implications for immunotherapy. Oncotarget. 2016;7:3403-15. PMID: 26655088.

19 Iwasaki T, Matsushita M, Nonaka D, Kuwamoto S, Kato M, Murakami I, et al. Comparison of Akt/mTOR/4E-BP1 pathway signal activation and mutations of PIK3CA in Merkel cell polyomavirus-positive and Merkel cell polyomavirus-negative carcinomas. Hum Pathol. 2015;46:210-6. PMID: 25466966.

20 Cimino PJ, Robirds DH, Tripp SR, Pfeifer JD, Abel HJ, Duncavage EJ. Retinoblastoma gene mutations detected by whole exome sequencing of Merkel cell carcinoma. Mod Pathol. 2014;27:1073-87. PMID: 24406863.

21 Alexandrov LB, Nik-Zainal S, Wedge DC, Aparicio SA, Behjati S, Biankin AV, et al. Signatures of mutational processes in human cancer. Nature. 2013;500:415-21. PMID: 23945592. 